\title{
Fibronectin mediates activation of stromal fibroblasts by SPARC in endometrial cancer cells
}

Sachiko Yoshida, Kazuo Asanoma * D, Hiroshi Yagi, Ichiro Onoyama, Emiko Hori, Yumiko Matsumura, Kaoru Okugawa, Hideaki Yahata and Kiyoko Kato

\begin{abstract}
Background: Matricellular glycoprotein, SPARC is a secreted molecule, that mediates the interaction between cells and extracellular matrix. SPARC functions as a regulator of matrix organization and modulates cell behavior. In various kinds of cancer, strong SPARC expression was observed in stromal tissues as well as in cancer epithelial cells. The function of SPARC in cancer cells is somewhat controversial and its impact on peritumoral stromal cells remains to be resolved.

Methods: We investigated the effects of SPARC expression in endometrial cancer cells on the surrounding stromal fibroblasts using in vitro co-culture system. Changes in characteristics of fibroblasts were examined by analysis of fibroblast-specific markers and in vitro contraction assay.

Results: SPARC induced AKT phosphorylation and epithelial-to-mesenchymal transition, consistent with previous reports. Cancer-associated fibroblasts of endometrial cancer expressed higher levels of mesenchymal- and fibroblast-associated factors and had a stronger contraction ability. Unexpectedly, cancer-associated fibroblasts expressed comparable levels of SPARC compared with fibroblasts from normal endometrium. However, co-culture of normal fibroblasts with SPARC-expressing Ishikawa cells resulted in activation of the fibroblasts. Immunodepletion of SPARC did not affect the activation of fibroblasts.

Conclusions: Our data indicated that SPARC activated fibroblasts only in the presence of fibronectin, which was abundantly secreted from SPARC-expressing endometrial cancer cells. These results suggested that a SPARCfibronectin-mediated activation of fibroblasts might be involved in enhanced mobility and invasion of cancer cells.
\end{abstract}

Keywords: Cancer-associated fibroblasts, Endometrial neoplasms, Extracellular matrix, FN1, SPARC

\section{Background}

The matricellular glycoprotein, SPARC is a secreted molecule that mediates interactions between the cell and extracellular matrix. SPARC functions as a regulator of matrix organization and modulates cell behavior $[1,2]$. Various functions of SPARC in cancer cells have been reported. In addition to modulation of extracellular

\footnotetext{
* Correspondence: asanoma@med.kyushu-u.ac.jp
}

Department of Obstetrics and Gynecology, Faculty of Medical Sciences, Kyushu University, 3-1-1 Maidashi, Higashi-ku, Fukuoka 812-8582, Japan matrix, SPARC also regulates cell adhesion, proliferation, survival, apoptosis, migration, invasion, and induction of epithelial-to-mesenchymal transition (EMT) in cancer cells $[1,3-5]$.

In addition to functions in cancer cells, SPARC also plays a critical role in stromal cells in in cancer progression. In various kinds of cancer, strong SPARC expression was observed in stromal cells in contrast with its low expression in cancer epithelial cells [6-9]. While SPARC secreted from stromal fibroblasts was suggested

(c) The Author(s). 2021 Open Access This article is licensed under a Creative Commons Attribution 4.0 International License, which permits use, sharing, adaptation, distribution and reproduction in any medium or format, as long as you give appropriate credit to the original author(s) and the source, provide a link to the Creative Commons licence, and indicate if changes were made. The images or other third party material in this article are included in the article's Creative Commons licence, unless indicated otherwise in a credit line to the material. If material is not included in the article's Creative Commons licence and your intended use is not permitted by statutory regulation or exceeds the permitted use, you will need to obtain permission directly from the copyright holder. To view a copy of this licence, visit http://creativecommons.org/licenses/by/4.0/ The Creative Commons Public Domain Dedication waiver (http://creativecommons.org/publicdomain/zero/1.0/) applies to the data made available in this article, unless otherwise stated in a credit line to the data. 
to suppress cancer cell proliferation or migration in vitro, SPARC expression in peritumoral fibroblasts correlated with worse prognosis in pancreatic cancer [8-10].

The effects of SPARC in host tissues have been studied by transplantation of cancer cells into SPARC-deficient mice. While murine mammary cancer cells transplanted in SPARC-deficient mice formed smaller tumors compared with controls, murine pancreatic cancer cells, Lewis lung cancer cells and lymphoma cells formed larger tumors and increased metastasis in the mice [11-15]. Mouse carcinogenesis models in a SPARC-deficient background have been also studied. Prostate and bladder carcinogenesis is enhanced in SPARC-deficient mice [16, 17]. However, other studies showed that skin squamous cell carcinoma and intestinal tumors were suppressed in SPARC-deficient mice $[18,19]$. Another report of SPARCdeficient mice did not find any changes in the cancer progression and metastasis in prostate and mammary carcinogenesis [20].

Therefore, the function of SPARC in oncogenesis is somewhat controversial and it cannot be determined based only on the endogenous expression of SPARC in cancer cells. Other factors including interactions with tumor microenvironment including extracellular matrix, stromal cells or proteolysis of SPARC may be involved but the mechanism remains largely unknown [1, 21-23].

In our previous study, we found that SPARC was exclusively expressed in endometrial cancer (EC) stem-like cells. EC cells with forced expression of SPARC formed tumors with larger amounts of peritumoral stromal tissue after subcutaneous inoculation in nude mice [24]. We also observed that while SPARC expression rate was low (28\%) in well differentiated endometrioid carcinoma grade 1 cases, its expression was enhanced in poorly differentiated endometrioid carcinoma, grade 3 (60\%), serous carcinoma (50\%) and clear cell carcinoma cases (73\%), which were thought to have aggressiveness [24]. However, the impact of the SPARC expression on the association between tumor cells and the adjacent stromal cells has not been well determined.

\section{Methods}

\section{Fibroblast isolation and cell culture}

Normal fibroblasts (NF) were isolated from six patients who underwent fertility treatment at the IVF Nagata Clinic (Table S1) [25]. Endometrial tissues were digested with $0.25 \%$ collagenase type I solution (Thermo Fisher Scientific, Inc., Waltham, MA, USA) for $2 \mathrm{~h}$ at $37^{\circ} \mathrm{C}$ using a rotating mixer. The cells were passed through $35 \mu \mathrm{m}$ cell strainer (Corning, Kennebunk, ME, USA), and suspended in Dulbecco's modified Eagle's medium (DMEM, Nacalai Tesque, Kyoto, Japan) supplemented with 10\% fetal bovine serum (FBS; Merck KGaA, Darmstadt, Germany), and $1 \%$ penicillin/streptomycin
(Nacalai Tesque). The cells were cultured at $37^{\circ} \mathrm{C}$ at $5 \%$ $\mathrm{CO}_{2}$ atmosphere. Cancer associated fibroblasts (CAF) were isolated from seven patients who underwent surgery at the Department of Obstetrics and Gynecology at Kyushu University Hospital (Table S2). Cancer tissues were minced and digested $2 \mathrm{mg} / \mathrm{mL}$ collagenase A (100 $\mathrm{mg} / \mathrm{ml}$, Sigma Aldrich, St. Louis, MO, USA) with DMEM containing $10 \% \mathrm{FBS}$ for $20 \mathrm{~min}$ at $37^{\circ} \mathrm{C}$. The cells were passed through a $40-\mu \mathrm{m}$ cell strainer (Corning) and cultured in DMEM with 10\% FBS and 1\% penicillin/streptomycin. NF and CAF at passage $3 \neg 8$ were used for experiments as were chosen also in other studies [26-31]. Written informed consent was obtained from all patients. The study was approved by the Ethical Committee of Kyushu University. Ishikawa EC cells were purchased from Japanese Collection of Research Bioresources (JCRB, Tokyo, Japan), and maintained in DMEM supplemented with $10 \%$ FBS and $1 \%$ penicillin/streptomycin. Ishikawa cells were used within 6 months of receipt.

\section{Reagents}

The AKT inhibitor, MK2206 (Selleck Chemicals, Houston, TX, USA) was used on Ishikawa cells at the indicated concentrations. Cells treated with MK2206 for 24 $\mathrm{h}$ were used for immunoblotting. For migration assays, MK2206 was added to both the upper and the lower chambers. Recombinant human SPARC was purchased from PeproTec Inc. (Rocky Hill, NJ, USA). One hundred $\mathrm{ng} / \mathrm{mL}$ of recombinant SPARC was used on fibroblasts unless otherwise indicated. Fibronectin (FN1) was purchased from Corning, and plates were coated with FN1 at a concentration of $50 \mu \mathrm{g} / \mathrm{mL}$.

\section{Lentivirus vector transduction}

FLAG-tagged SPARC plasmid was kindly provided by Dr. Sasaki Takako at Oita University, and was ligated into a pLX302 vector. An empty pLX302 vector was used as a control. Lentivirus vectors were produced as described previously [32]. To generate stable cell lines, lentivirus-infected Ishikawa cells were cultured in $2 \mu \mathrm{g} /$ $\mathrm{mL}$ of puromycin.

\section{Collagen gel contraction assay}

Collagen lattices were prepared using Cellmatrix (Nitta Gelatin Co., Osaka, Japan), sterile reconstitution buffer (50 mM NaOH, 2.2\% $\mathrm{NaHCO}_{3}, 200 \mathrm{mM} \mathrm{HEPES}$ ) and $5 \times$ DMEM (Nissui Pharmaceutical Co., Tokyo, Japan) mixed at a ratio of 7:2:1. NF were suspended in the collagen solution $\left(2 \times 10^{5}\right.$ cells $\left./ \mathrm{ml}\right)$. Next $300 \mu \mathrm{L}$ of the mixture was poured into 24-wells plates, and the mixture was allowed to polymerize for $30 \mathrm{~min}$ at $37^{\circ} \mathrm{C}$. After polymerization, $300 \mu \mathrm{L}$ serum-free DMEM was added to the collagen gel. After $24 \mathrm{~h}$, the collagen gel was released 
mechanically and the area of the gel was measured $24 \mathrm{~h}$ later. The contraction ratio (\%) was calculated as (well area - gel area) / (well area) $\times 100$.

\section{Immunohistochemistry}

Paraffin sections were deparaffinized, and rehydrated. The slides were washed in PBS after applying Proteinase K (Dako, Carpinteria, CA, USA) for $6 \mathrm{~min}$ and blocked in Protein Block Serum Free (DAKO) for $30 \mathrm{~min}$ at room temperature. The slides were immunolabeled with primary antibodies against SPARC (1:500, M125, TAKA RA, Tokyo, Japan) and FN1 (1:300, ab2413, Abcam, Cambridge, UK) overnight at $4{ }^{\circ} \mathrm{C}$ after incubation with Antibody Diluent Solution (DAKO). The slides were then rinsed with PBS and incubated with N-Histofine Simple Stain MAX PO (Nichirei, Tokyo, Japan) at room temperature for $30 \mathrm{~min}$. Visualization of the immunoreaction was carried out by incubation with 3,3-diaminobenzidine (DAB). Finally, sections were counterstained with hematoxylin. Images were captured using Olympus Microscope BX53 and cellSens imaging software (Olympus, Tokyo, Japan). The images were acquired at a resolution of 600dpi.

\section{Immunocytochemistry}

Fibroblasts were seeded into 8-well Lab TekII chamber slides (Thermo Fisher Scientific) at a density of $1 \times 10^{5}$ cells per chamber. After $24 \mathrm{~h}$ incubation, the cells were fixed in $4 \%$ paraformaldehyde for $15 \mathrm{~min}$, permeabilized with $0.25 \%$ Triton -100 in PBS for $10 \mathrm{~min}$, and blocked with Protein Block Serum Free (Dako) for $10 \mathrm{~min}$ at room temperature. The cells were incubated at $4{ }^{\circ} \mathrm{C}$ overnight with primary antibodies against VIM (1:200, sc-6260, Santa Cruz Biotechnology, Santa Cruz, CA, USA) and pan-Cytokeratin (1:200, sc-8018, Santa Cruz Biotechnology). The cells were incubated at room temperature for $30 \mathrm{~min}$ with a secondary antibody, Alexa Fluor 488 goat anti-mouse IgG (1:500, Life Technologies, Waltham, MA, USA). Cells were mounted in ProLong Gold antifade reagent with 4',6-diamidino-2-phenylindole (DAPI) (Invitrogen, Carlsbad, CA, USA) and visualized by BZ-X710(KEYENCE Corporation, Osaka, Japan).

\section{Immunoblotting}

Cells were lysed in CelLyticM (Sigma Aldrich) with protease inhibitor cocktail (Sigma Aldrich) and phosphatase inhibitor cocktail (Nacalai Tesque). Equal amounts of lysate were separated by $10 \%$ sodium dodecyl sulfate polyacrylamide gel electrophoresis (SDS-PAGE) and transferred to a polyvinylidene difluoride membrane (Millipore, Bedford, MA, USA). The membranes were blocked with $5 \%$ milk or Blocking One-P (Nacalai Tesque) for $20 \mathrm{~min}$, and then incubated at $4{ }^{\circ} \mathrm{C}$ overnight with primary antibodies against ACTA2 (1:1000, A2547,
Sigma-Aldrich), FAP (1:500; ab28244, Abcam), VIM (1: 1000, sc-6260, Santa Cruz Biotechnology), CDH2 (1: 1000, sc-7939, Santa Cruz Biotechnology), CDH1 (1: 1000, \#3195, Cell Signaling Technology, Beverly, MA, USA), SPARC $(0.75 \mu \mathrm{l} / \mathrm{mL}$, AON-5031, Haematologic Technologies Inc., Essex Junction, VT, USA), FN1 (1: 1000, ab23750, Abcam), GAPDH (1:1000, sc-25,778, Santa Cruz Biotechnology), ACTB (1:1000, sc-81,178, Santa Cruz Biotechnology), FLAG M5 (1:5000, F4042, Sigma-Aldrich), phosphorylated-AKT (Ser473, 1:1000, \#9271, Cell Signaling Technology) and AKT (1:1000, \#9272, Cell Signaling Technology). The membranes were incubated at room temperature for $1 \mathrm{~h}$ with the appropriate secondary antibody, mouse or rabbit IgGPeroxidase (Sigma-Aldrich). The blots were processed using enhanced chemiluminescence and bands were detected using a ChemiDoc XRS Imaging System (Bio Rad, Richmond, CA, USA).

\section{Elisa}

After NF and Ishikawa cells were incubated with serumfree DMEM for $24 \mathrm{~h}$, the culture medium was collected. The concentrations of SPARC and FN1 were analyzed using enzyme-linked immunosorbent assays (SPARC, DSP00; FN1, DFBN10, R\&D Systems, Minneapolis, MN, USA) according to the manufacturer's instructions.

\section{Real-time reverse transcription polymerase chain reaction (RT-qPCR)}

Total RNA was extracted from cells using RNeasy Mini Kits (Qiagen, Valencia, CA, USA), and cDNA was generated by ReverTra Ace- $\alpha$ (Toyobo, Osaka, Japan). RealTime RT-PCR was performed using TaqMan primers and primers for SPARC (Hs00234160_m1), FN1 (Hs00365052_m1), VIM (Hs00185584_m1), CDH2 (Hs00983056_m1), and CDH1 (Hs01023894_m1) provided by Thermo Fisher Scientific (Waltham, MA, USA). qPCR was performed using TaqMan Fast Advanced Master Mix and StepOnePlus system (Thermo Fisher Scientific). Gene expression levels were obtained using individual values normalized to $18 \mathrm{~S}$ rRNA (Hs99999901_s1, Thermo Fisher Scientific).

\section{Migration assay}

Cells $\left(2.5 \times 10^{5}\right)$ suspended in serum-free medium were plated on a Falcon cell culture insert with $8.0 \mu \mathrm{m}$ pores (Corning). DMEM with 10\% FBS was placed in the lower wells. After $48 \mathrm{~h}$, the membranes were collected and stained with Diff-Quick staining solutions (Sysmex Corp., Kobe, Japan).

\section{Knockdown of FN1 gene expression in Ishikawa cells}

A validated short interfering RNA for FN1 (siFN1; siGENOME Human FN1(2335) siRNA-SMART pool) 
and negative control siRNA (siGENOME Non-Targeting siRNA pool\#2: Dharmacon) were transfected into SPAR C-expressing Ishikawa cells using Lipofectamine 2000 (Thermo Fisher Scientific). Forty-eight hours after transfection, the cells were used for immunoblotting or cultured in serum-free medium for another $48 \mathrm{~h}$ for production of conditioned medium.

\section{Immunodepletion of FLAG-tagged SPARC in conditioned media}

Conditioned media from Ishikawa cells were incubated with anti-FLAG M2 affinity gel $(1: 300)$ at $4{ }^{\circ} \mathrm{C}$ with agitation overnight. The media were centrifuged at $5000 \mathrm{~g}$ for $2 \mathrm{~min}$ to remove debris.

\section{Co-culture of NF with Ishikawa cells}

NF were co-cultured with Ishikawa cells in a Falcon cell culture insert with $0.4 \mu \mathrm{m}$ pores (Corning, Kennebunk, $\mathrm{M})$. For collagen gel contraction assay, NF were collected after $24 \mathrm{~h}$ co-culture with Ishikawa cells and used for assays.

\section{Statistical analysis}

Data are represented as the means \pm standard deviation (SD) and were analyzed with two-sided Student's $t$-test. The data in Figs. 5d, e, g, h and 6c, d, g and h were analyzed with one-way ANOVA (analysis of variance) followed by Newman-Keuls test. $P$-values of less than 0.05 were considered statistically significant.

\section{Results}

SPARC expression in NF and CAF from EC

Cancer cells are closely associated with the surrounding stromal fibroblasts. A specific type of activated fibroblasts, CAF plays a critical role in cancer cells in creating the extracellular matrix and tumor microenvironment [33]. In our previous study, we found that abundant SPARC protein localized in tumor stroma is associated with an aggressive type of EC [24]. To explore the
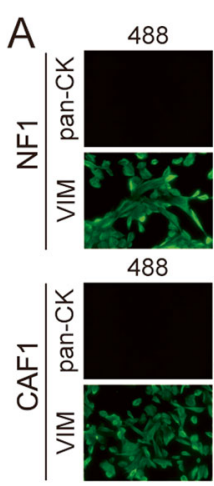

C
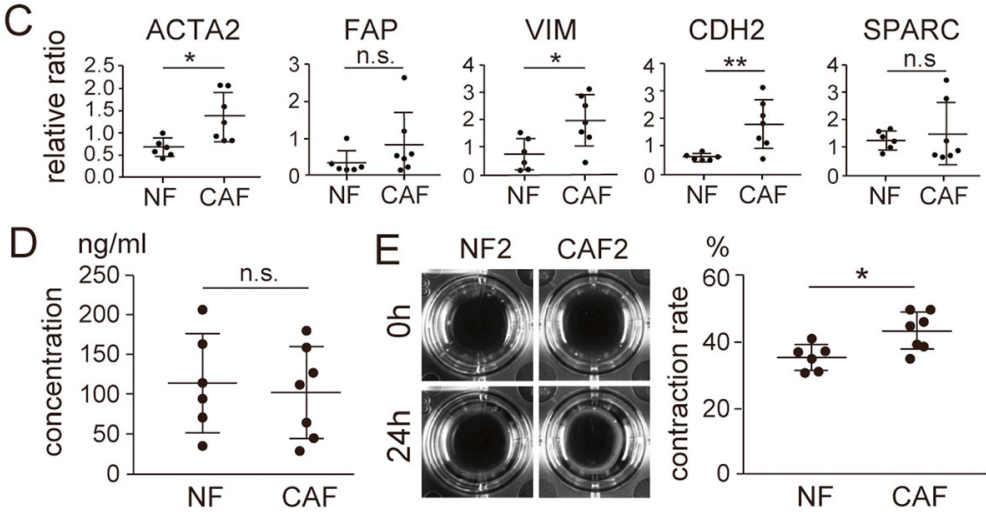

Fig. 1 Characteristics of isolated fibroblasts from normal endometrium and endometrial cancer specimens. (A) Fibroblasts isolated from normal endometrium sample \#1 (top, NF1) and those from endometrial cancer sample \#1 (bottom, CAF1) were used for immunocytochemistry. Anti-pan-cytokeratin (pan-CK) and anti-VIM antibodies were used to visualize protein expression. (B) Isolated NF $(n=6)$ and CAF lines $(n=7)$ were used for immunoblotting to detect the expression of ACTA2, FAP, VIM, CDH2 and SPARC. GAPDH was used as an internal control. Full-length blot images are presented in Supplementary Fig. 1. (C) Semi-quantification of the immunoblotting data in (B). Intensity of the bands was quantified using Image J (https://imagej.nih.gov/ij/). Values of the protein-of-interest were corrected using the intensity of GAPDH bands. (D) ELISA analysis of SPARC secreted from the NF and CAF lines. (E) In vitro contraction analysis of the NF and CAF lines. The images show representative results of the experiments. The graphs on the right show quantification data of the results. The scale bars indicate $100 \mu m$. 488 , Alexa Fluor 488 ; pan-CK, pan-cytokeratin; NF, normal fibroblasts; CAF, cancer-associated fibroblasts; n.s., not significant; ${ }^{*}, P<0.05$ 
impact of SPARC expression in CAF, we first compared the characteristics between NF from normal endometrium and CAF isolated from EC specimens. Successful isolation of fibroblasts from normal endometrium and EC specimens was confirmed by immunocytochemistry (Fig. 1a). All cells were positive for VIM expression and negative for pan-cytokeratin expression. As reported elsewhere [33], higher expression of markers, such as ACTA2, VIM and $\mathrm{CDH} 2$ for activated fibroblasts, was observed in CAF from EC compared with NF (Fig. 1b and c; Supplementary Fig. 1). Unexpectedly, the expression and secretion of SPARC was comparable between NF and CAF (Fig. 1b-d; Supplementary Fig. 1). We also evaluated the functional activity of fibroblasts by a collagen gel contraction assay. As expected, CAF showed a higher contraction rate than NF (Fig. 1e). These results suggested that the abundant SPARC protein localized in the stroma of aggressive EC specimens was not produced by the stromal fibroblasts.
Forced expression of SPARC in EC cells enhanced EMT and cell mobility mediated by AKT activation

Next, we studied the impact of SPARC expression in EC cells. Our previous study showed that SPARC expression was absent in Ishikawa cells [24]. We confirmed successful expression and secretion of SPARC in Ishikawa cells by lentivirus-mediated expression (Fig. 2a-c, Supplementary Fig. 2). Forced expression of SPARC in Ishikawa cells induced the protein and mRNA expression of mesenchymal markers CDH2, VIM and FN1 (Fig. 2d and e; Supplementary Fig. 3). In our previous study, we already reported that SPARC-expressing Ishikawa cells expressed remarkably high amounts of FN1 [24]. Consistent with the induction of EMT, cell mobility was enhanced in SPARC-expressing Ishikawa cells (Fig. 2f). SPARC expression in melanoma and non-small cell lung cancer cells was reported to induce EMT mediated by AKT phosphorylation $[4,5]$. We found that AKT phosphorylation (Ser473) was induced in Ishikawa cells by

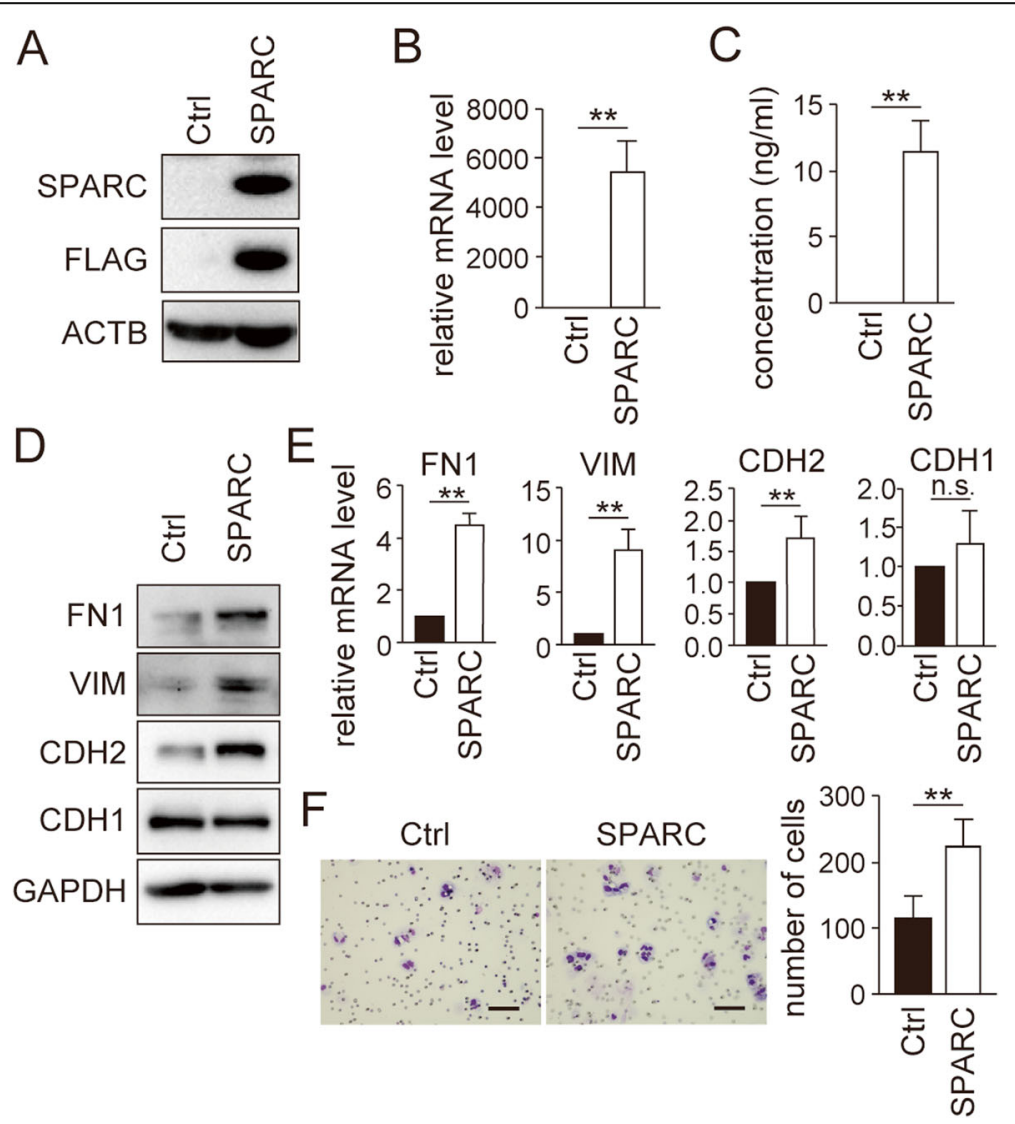

Fig. 2 Forced expression of SPARC in Ishikawa cells induces EMT and cell migration. Successful lentiviral transduction of SPARC in Ishikawa cells was confirmed using immunoblotting (A), RT-qPCR (B) and ELISA (C). ACTB was used as an internal control (A). (D) The expression of FN1, VIM, CDH2 and CDH1 protein and mRNA in SPARC-expressing Ishikawa cells was analyzed using immunoblotting (D) and RT-qPCR (E). (F) In vitro cell migration was analyzed using transwell chamber assays. Images on the left show representative results. Graphs on the right show quantification data of the results. The scale bars indicate $100 \mu \mathrm{m}$. Ctrl, control; n.s., not significant; ${ }^{*}, P<0.05 ;{ }^{*}, P<0.01$. Full-length blot images are presented in Supplementary Fig. 2 and 3 (A, D). The experiments were independently repeated three times and representative data were shown 
SPARC expression (Fig. 3a; Supplementary Fig. 4). As expected, the AKT inhibitor MK2206 canceled the induction of phosphorylated AKT by SPARC expression in a dose-dependent manner (Fig. 3b; Supplementary Fig. 5). Notably, MK2206 also suppressed the expression of CDH2, VIM and FN1 and the cell mobility induced by SPARC expression (Fig. 3b and c; Supplementary Fig. 5). These results suggested that the enhanced EMT and cell mobility by SPARC expression was mediated by AKT signal activation.

\section{SPARC-expressing EC cells are responsible for the} activation of fibroblasts but SPARC alone is not sufficient for the activation

We next examined whether SPARC expression in EC cells affected nearby fibroblasts. NF were co-cultured with control or SPARC-expressing Ishikawa cells separated by a membrane (Fig. 4a). SPARC expression in Ishikawa cells enhanced the expression of ACTA2 and $\mathrm{CDH} 2$ in the fibroblasts and cell proliferation (Fig. 4b and c; Supplementary Fig. 6). SPARC expression in Ishikawa cells also functionally activated NF; the contraction ability was enhanced in NF co- cultured with SPARC-expressing Ishikawa cells (Fig. $4 d)$. These results suggested that the fibroblasts were activated by a factor secreted from SPARC-expressing EC cells. We thus first examined the impact of SPARC secreted from SPARC-expressing cells on NF. An anti-FLAG antibody was used to immunodeplete FLAG-tagged SPARC from conditioned medium collected from FLAG-SPARC-expressing cells (Fig. 5a). Most of the SPARC was successfully depleted from conditioned media (Fig. 5b; Supplementary Fig. 7). However, the inhibition of ACTA2 and $\mathrm{CDH} 2$ by SPARC depletion was only modest (Fig. $5 \mathrm{c}$; Supplementary Fig. 8). The cell proliferation and contraction rate of NF were also not drastically altered by SPARC depletion (Fig. $5 \mathrm{~d}$ and e). In the further experiments, recombinant SPARC was added to the culture medium of NF. As predicted, recombinant SPARC at the various concentrations examined did not affect the expression of ACTA2 and CDH2, cell proliferation, and contraction ability (Fig. 5f-h; Supplementary Fig. 9). These results suggested that a factor other than SPARC itself secreted from SPARC-expressing EC cells was involved in mediating the effects on NF.
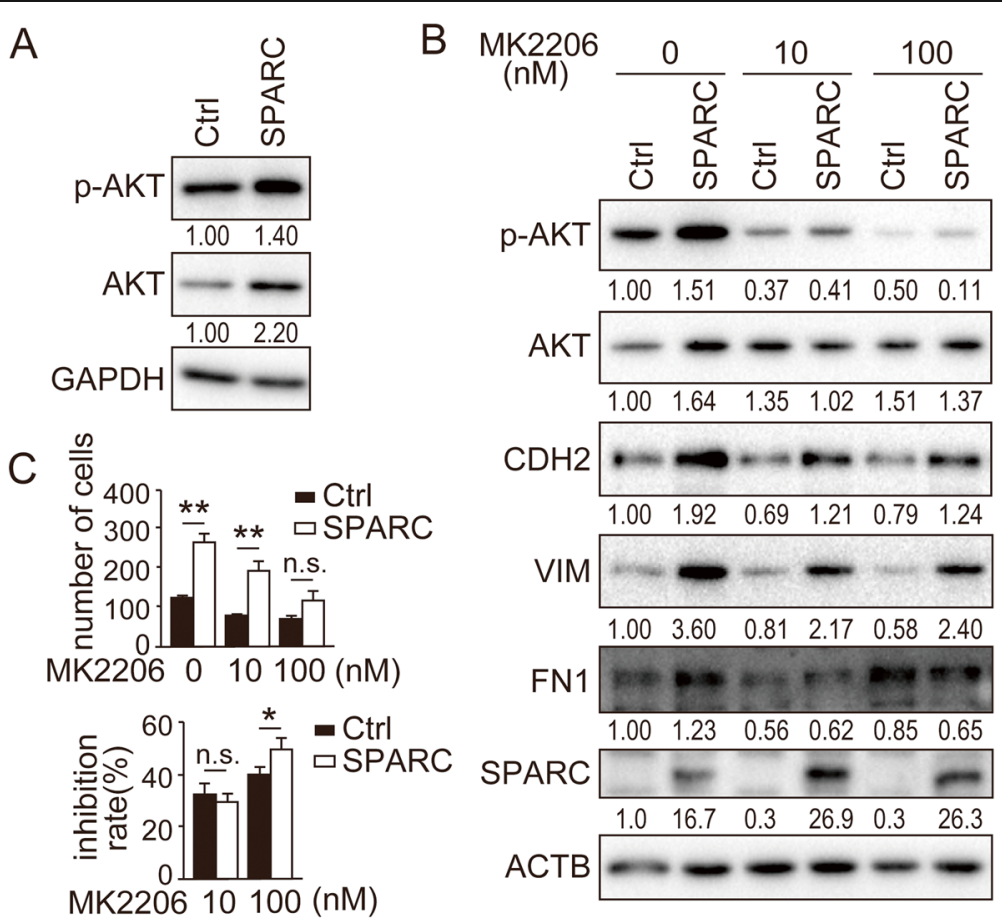

Fig. 3 Induction of EMT and cell migration by SPARC is mediated by the AKT pathway. (A) Immunoblotting analysis of AKT and phosphorylated-AKT in SPARC-expressing Ishikawa cells. (B) Immunoblotting analysis of SPARC-expressing Ishikawa cells treated with indicated concentrations of the highly selective AKT inhibitor, MK2206. Antibodies against AKT, phosphorylated-AKT ( $p-A K T$, Ser473), CDH2, VIM, FN1 and SPARC were used for immunoblotting. (A, B) Intensity of the bands was quantified using Image J. Values of the protein-of-interest were corrected using the intensity of GAPDH and ACTB bands, respectively. (C) In vitro cell migration of SPARC-expressing Ishikawa cells treated with indicated concentrations of MK2206. Numbers of migrated cells are shown in the top graph. Inhibition ratio of migrated cells compared with numbers of migrated cells at $0 \mathrm{nM}$ is shown in the bottom graph. Ctrl, control; n.s., not significant; ${ }^{*}, P<0.05 ;{ }^{*}, P<0.01$. Full-length blot images are presented in Supplementary Fig. 4 and 5 (A, B). The experiments were independently repeated three times and representative data were shown 

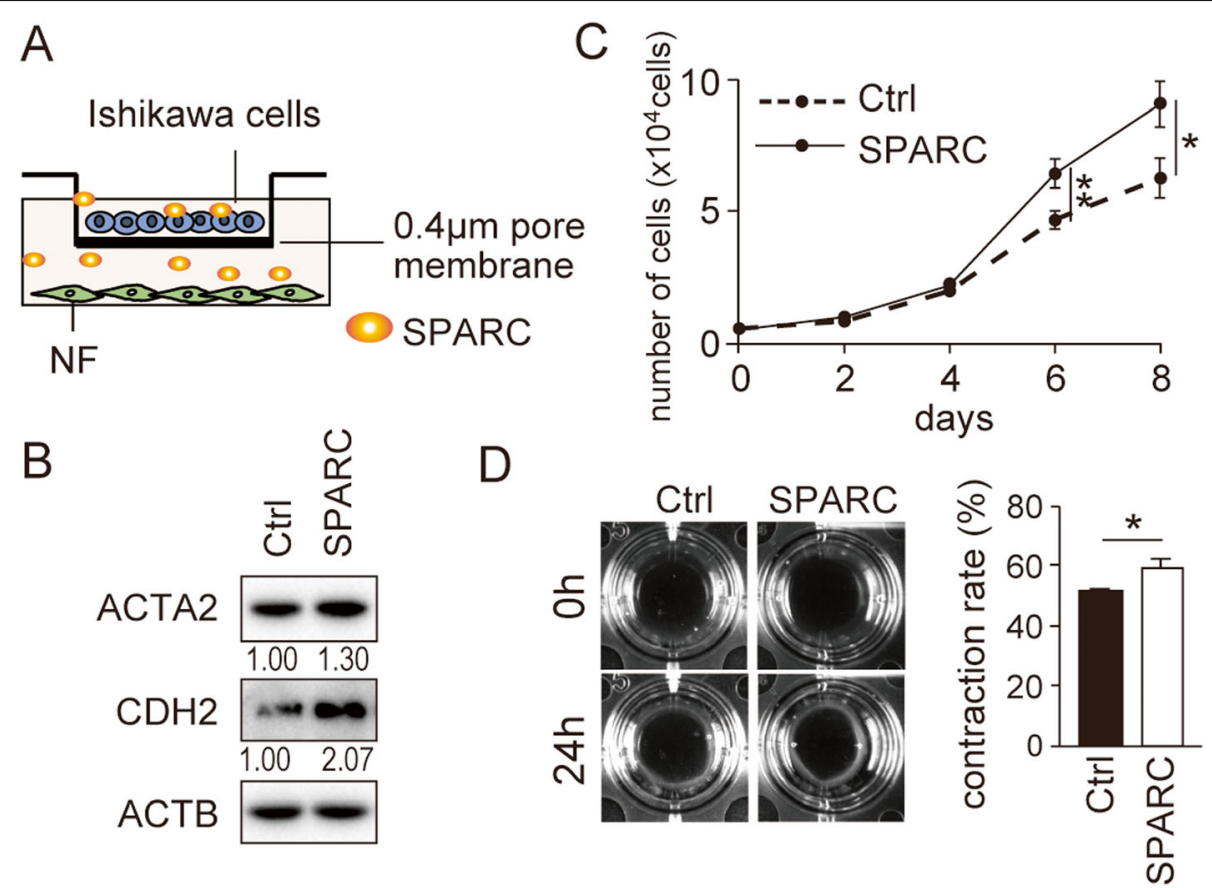

Fig. 4 Co-culture of SPARC-expressing Ishikawa cells activates normal fibroblasts. (A) Schematic presentation of co-culture experiment of normal fibroblasts (NF) with SPARC-expressing Ishikawa cells. (B) Immunoblotting analysis of ACTA2 and CDH2 in NF co-cultured with SPARCexpressing Ishikawa cells. Intensity of the bands was quantified using Image J. Values of the protein-of-interest were corrected using the intensity of ACTB bands. Full-length blot images are presented in Supplementary Fig. 6. Cell proliferation (C) and in vitro contraction ability (D) of NF were also analyzed. A representative result is shown in the left panels. The right graph shows quantification data of the results. NF, normal fibroblasts; $\mathrm{Ctrl}$, control; ${ }^{*}, P<0.05 ;{ }^{*}, P<0.01$. The experiments were repeated using fibroblasts from three independent cases and representative data were shown

FN1 is required for activation of fibroblasts by SPARCexpressing EC cells

We next focused on FN1 because abundant FN1 was detected in SPARC-expressing Ishikawa cells compared with control cells (Fig. 2d; Supplementary Fig. 3). We observed higher amounts of FN1 secreted from SPARCexpressing Ishikawa cells compared with control cells (Fig. 6a). We next studied the impact of FN1 on NF. NF on an FN1-coated dish showed elevated expression of ACTA2 and CDH2 (Fig. 6b; Supplementary Fig. 10). Furthermore, addition of recombinant SPARC on NF on the FN1-coated dish enhanced the cell proliferation and contraction ability of NF (Fig. 6c and d). To study the effect of FN1 on NF, we performed knockdown experiments of FN1 in SPARC-expressing Ishikawa cells. Successful siRNA-mediated reduction of FN1 secreted from SPARC-expressing Ishikawa cells was confirmed (Fig. 6e). NF cultured in conditioned media from SPAR C-expressing Ishikawa cells in which FN1 was knocked down showed reduced expression of CDH2 (Fig. 6f; Supplementary Fig. 11). In addition, SPARC depletion and FN1 knockdown in SPARC-expressing Ishikawa cells remarkably suppressed the cell proliferation and contraction ability of NF (Fig. $6 \mathrm{~g}$ and $\mathrm{h}$ ). These results suggested that FN1 is necessary for the activation of fibroblasts by SPARC in the extracellular matrix. Both FN1 and SPARC abundantly secreted from SPARC-expressing EC cells might be necessary for full activation of fibroblasts.

SPARC expression in cancer cells is adjacent to FN1 expression in the surrounding stromal tissue

Immunohistochemical analysis of EC specimens showed that SPARC expression level was stronger in the cancer cells compared with the stromal area (Fig. 7a-f). This result suggested that SPARC expression and secretion from EC cells affected stromal fibroblasts. Analysis of FN1 expression in the sequential sections showed that SPARC expression in cancer cells was adjacent to FN1 expression in the surrounding stromal tissue (Fig. $7 \mathrm{a}-\mathrm{f}$ ). In some cases, SPARC expression was exclusive in some part of the cancer tissue. The region of cancer tissue positive for SPARC also showed FN1 expression in the stromal area, and the region without SPARC expression also lacked FN1 expression the stromal area (Fig. 7e-h). The above results suggested that FN1 secreted from SPARC-expressing cancer cells pooled in the surrounding stroma to activate the stromal fibroblasts. 


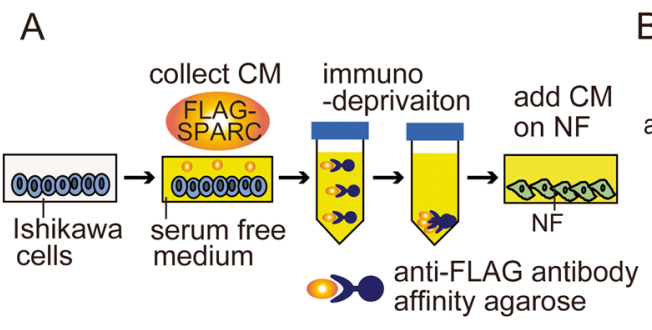

B

C

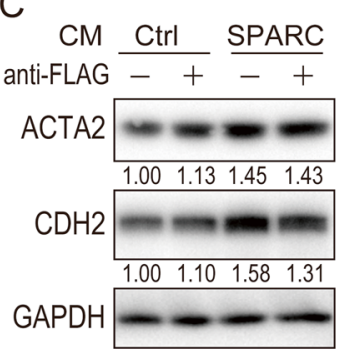

$\mathrm{F}$

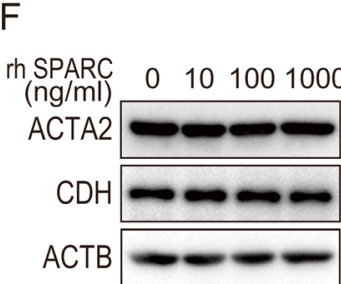

D

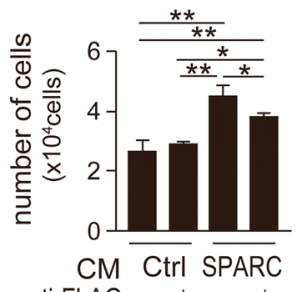

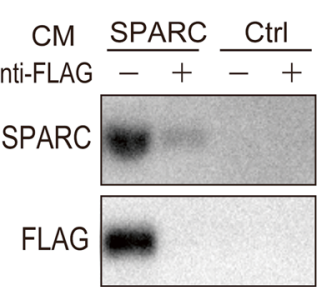

E

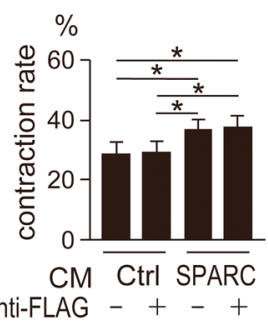

G

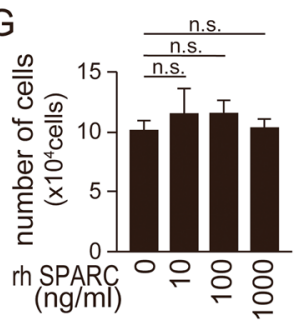

$\mathrm{H}$

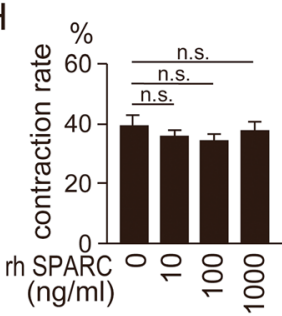

Fig. 5 Deprivation of SPARC in conditioned media from SPARC-expressing Ishikawa cells still activates normal fibroblasts. (A) Schematic presentation of the production of conditioned media from SPARC-expressing Ishikawa cells immunodepleted of SPARC using anti-FLAG antibody. (B) Successful immunodepletion of SPARC in conditioned media from control and SPARC-expressing Ishikawa cells was confirmed using immunoblotting. (C) Protein expression of ACTA2 and CDH2 in NF cultured in the conditioned media with or without immunodepletion of SPAR C. Intensity of the bands was quantified using Image J. Values of the protein-of-interest were corrected using the intensity of GAPDH bands. NF were also analyzed for cell proliferation on day 6 (D) and in vitro contraction (E) in the conditioned media. (F-H) NF were treated with the indicated concentration of recombinant SPARC. Protein expression of ACTA2 and CDH2 (F), cell number on day 6 (G) and in vitro contraction ability $(H)$ were assessed. NF, normal fibroblasts; Ctrl, control; rh SPARC, recombinant human SPARC; CM, conditioned media; n.S., not significant; *, $P<0.05$; **, $P<0.01$. Full-length blot images are presented in Supplementary Fig. 7, 8 and 9 (B, C, F). The experiments were independently repeated three times using identical fibroblasts and representative data were shown

\section{Discussion}

Here we showed that SPARC expression in Ishikawa EC cells induced EMT, cell invasion and AKT phosphorylation. The allosteric inhibitor of AKT inhibited EMT and cell migration (Figs. 2 and 3). These results were consistent with previous studies that showed that SPAR $\mathrm{C}$ induces AKT phosphorylation, which is important for induction of EMT, cell survival, anti-apoptotic activity of SPARC $[4,34,35]$.

In addition to the expression of SPARC in cancer cells, its expression in stromal cells also plays a critical role in cancer progression $[6,10,36,37]$. However, our study showed no remarkable differences in SPARC expression between CAF and NF. In most fibroblasts including NF, the expression level of SPARC was rather high (Fig. 1b and c; Supplementary Fig. 1). In our co-culture assay system of fibroblasts with SPARC-expressing Ishikawa cells, activation of fibroblast-specific gene expression and function was observed (Fig. 4). We observed enhanced proliferation of NF co-cultured with the SPARCexpressing cancer cells in vitro (Fig. 4c). The result may explain why larger amounts of stromal tissues were reported to accompany SPARC-expressing cancer cells in vivo $[24,38,39]$. These results suggested that SPARC expression in fibroblasts was not critical for fibroblast activation, but some factors secreted from SPARC-expressing Ishikawa cells were involved in this activation. Our immunodepletion experiment showed that SPARC itself did not affect fibroblast activation (Fig. 5). Moreover, exogenous addition of recombinant SPARC also did not affect fibroblast activation (Fig. 5). The recombinant SPARC we used was produced in $\mathrm{CHO}$ cells and its biological activity was confirmed by osteoblast differentiation of MC3T3 cells at a concentration of 0.5$0.7 \mu \mathrm{g} / \mathrm{mL}$ (PeproTec Inc. Rocky Hill, NJ, USA). Several studies used concentrations of approximately $1.0 \mu \mathrm{g} / \mathrm{mL}$ 

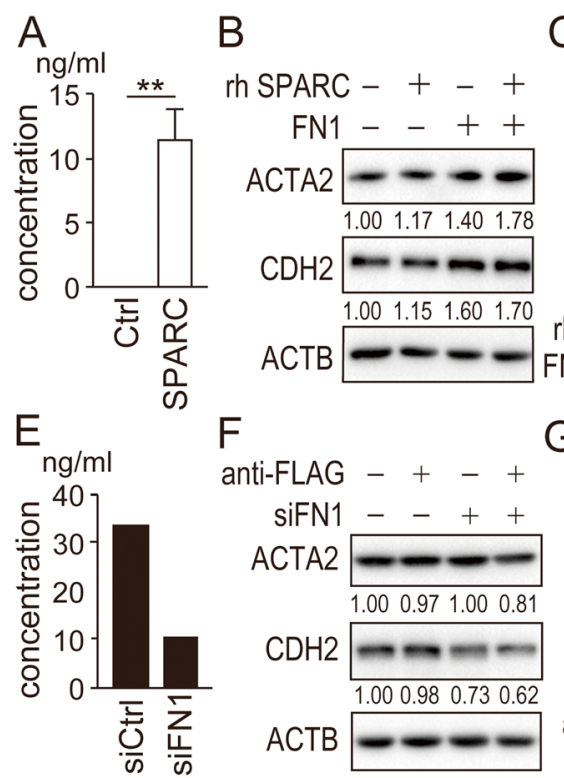

C

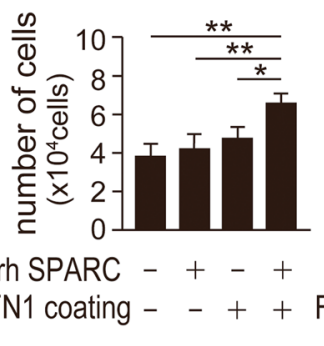

G

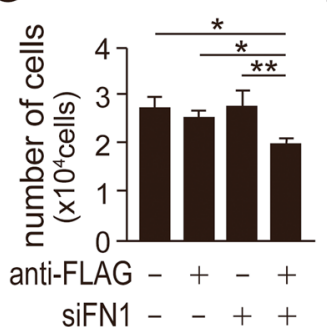

$\mathrm{D}$

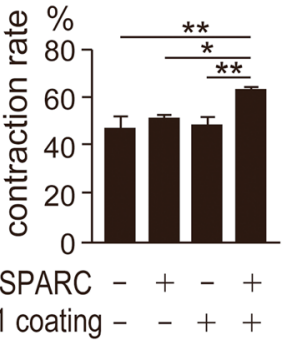

$\mathrm{H}$

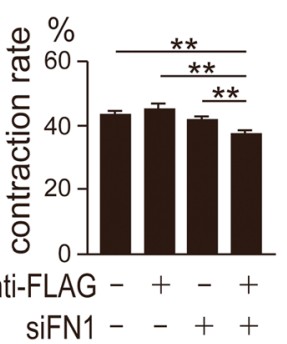

Fig. 6 FN1 secreted from SPARC-expressing Ishikawa cells activates normal fibroblasts. (A) Amount of FN1 secreted from SPARC-

expressing Ishikawa cells was measured by ELISA. (B) Protein levels of ACTA2 and CDH2 in NF cultured on FN1-coated dishes in the presence of recombinant SPARC were analyzed using immunoblotting. (C) NF were also analyzed for cell proliferation on day 6 in the same culture condition as (B). (D) NF cultured in the same condition as (B) were moved to collagen gel for in vitro contraction assays. (E) Successful knockdown of FN1 by siRNA (siFN1) was confirmed using ELISA. (F) Protein levels of ACTA2 and CDH2 in NF cultured in conditioned media from SPARC-expressing Ishikawa cells with siFN1 or immunodepleted of SPARC were analyzed using immunoblotting. NF were also analyzed for cell proliferation on day $6(\mathrm{G})$ and in vitro contraction $(\mathrm{H})$ in the conditioned media. (B, F) Intensity of the bands was quantified using Image J. Values of the protein-ofinterest were corrected using the intensity of ACTB bands. Ctrl, control; rh SPARC, recombinant human SPARC; siCtrl, control siRNA; *, $P<0.05$; **, $P<0.01$. Full-length blot images are presented in Supplementary Fig. 10 and 11 (B, F). The experiments were independently repeated three times using identical fibroblasts and representative data were shown

of recombinant SPARC [9, 40, 41]. We thus applied up to $1.0 \mu \mathrm{g} / \mathrm{mL}$ of recombinant SPARC in our experiments.

Although it was reported that fibroblasts in culture were different from freshly isolated fibroblasts from tissue in gene expression profile, we used fibroblasts passaged minimal times to exclude endothelial cells and epithelial cells [42-44]. Purification of fibroblasts was reported to obtained after at least three passages $[45,46]$. NF and $\mathrm{CAF}$ at passage $3 \neg 8$ were used for experiments as were chosen also in other studies [26-31].

Figure $5 \mathrm{~d}$ and e showed that immunodepletion of SPARC made only modest changes in fibroblast proliferation and contraction of the fibroblasts. In Fig. $6 \mathrm{~g}$ and $\mathrm{h}$, immunodepletion of SPARC again made modest changes in the proliferation and contraction. These

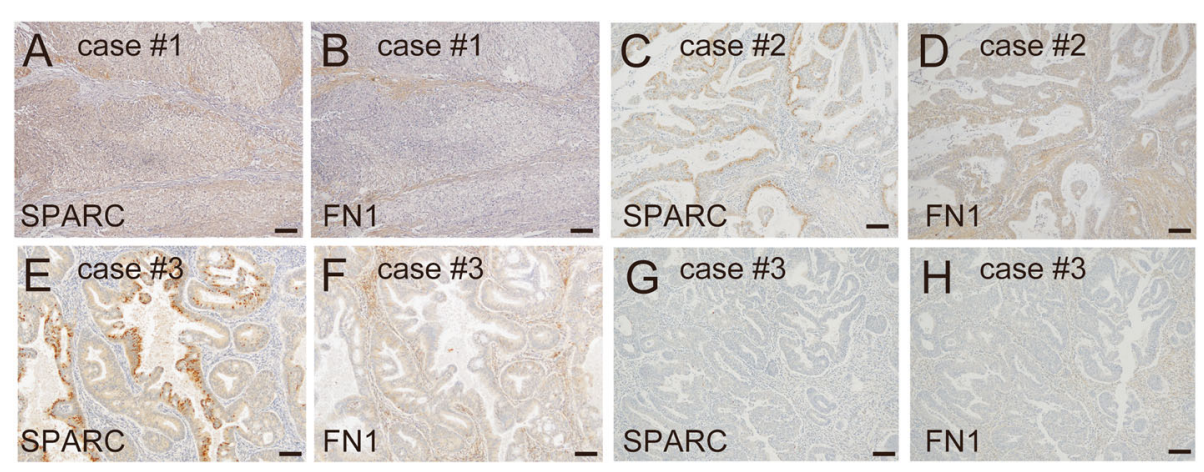

Fig. 7 SPARC expression sites were adjacent to areas with FN1 expression in endometrial cancer tissues. Three cases of endometrial cancer, (A, B) endometrioid carcinoma grade 3 at stage IA (case \#1), (C, D) endometrioid carcinoma grade 1 at stage IA (case \#2), and (E-H) endometrioid carcinoma grade 1 at stage IA (case \#3) were examined for SPARC (A, C, E, G) and FN1 (B, D, F, H) expression by immunohistochemistry. In the third case, a SPARC-positive area (E) and SPARC-negative area (G) were examined for FN1 expression ( $F, H)$. The scale bars indicate $100 \mu \mathrm{m}$ 
results were consistent. Next, Fig. $6 \mathrm{c}$ and d suggested that FN1 affected the proliferation and contraction only in the presence of SPARC. Considering this result, siFN1 was expected to reduce the proliferation and contraction regardless of SPARC. However, Fig. $6 \mathrm{~g}$ and $\mathrm{h}$ indicated that siFN1 made a difference only in the absence of SPARC. One possible explanation might be that conditioned media from siFN1 transfected cells still produced some amount of FN1 (Fig. 6e). This amount of FN1 might be enough to activate the fibroblast proliferation and contraction only in the presence of SPARC, but not in the absence of SPARC.

In our previous study, we studied the characteristics of EC stem-like cells isolated as side-population (SP) using fluorescence-activated cell sorting. SP fraction of HEC-1 cells expressed more than 7-fold higher amount of FN1 than non-SP fraction of HEC-1 cells [47]. SP HEC-1 cells also overexpressed SPARC compared to non-SP HEC-1 cells [24]. Furthermore, we observed forced expression of SPARC in EC cells induced FN1 expression and secretion (Figs. 2d,e, 3b, 6a ; Supplementary Fig. 3, 5) [24]. These data suggest that abundant FN1 expression in EC stem-like cells depend on overexpression of SPARC.

In support of the in vitro results, our immunohistochemical study showed that SPARC expression sites were adjacent to areas with FN1 expression (Fig. 7). The induction of FN1 by forced expression of SPARC in Ishikawa cells was suggested to mediate AKT activation because an AKT inhibitor suppressed FN1 expression (Fig. 3b; Supplementary Fig. 5). Our results showed that recombinant SPARC induced fibroblast activation only in the presence of FN1 (Figs. 5f-h and 6b-d; Supplementary Fig. 9, 10). SPARC was reported to be critical for FN1induced ILK activation and actin stress fiber formation in fibroblasts [48]. SPARC expression in cancer cells enhanced their migration and invasion on FN1-coated dishes $[49,50]$. Our results also suggested that the cooperation of SPARC and FN1 is critical for full activation of fibroblasts (Fig. 6b-d; Supplementary Fig. 10). A similar conclusion was obtained from our experiments using FN1 knockdown and SPARC depletion in SPARCexpressing Ishikawa cells (Fig. 6f-h; Supplementary Fig. 11). SPARC does not directly support cell attachment and is considered anti-adhesive. SPARC binds to several integral components of the ECM and exhibits an anti-adhesive effect that includes abrogation of focal adhesions and disruption of cell spreading and mobility $[51,52]$. SPARC might interact with FN1 to exhibit an anti-adhesive effect.

Activation of fibroblasts is determined not only by gene expression but also by a functional aspect. ECM reorganization and ECM stiffness is reported to be determined by changes in collagen cross-linking, which drives tumor progression through PI3K-AKT activation mediated by integrin receptors [53, 54]. SPARC was reported to interact with integrin $[9,14,55,56]$. Incorporation of SPARC-integrin-FN1-mediated activation of AKT may be involved in activation of fibroblasts and dominant changes in ECM to help cancer cells to mobilize or invade into adjacent stroma.

The limitation of this study is that the molecular mechanism of SPARC and FN1 for activation of fibroblasts remains to be clarified.

\section{Conclusions}

In conclusion, our study indicated that SPARC induced AKT phosphorylation and EMT in cancer cells, as reported elsewhere $[4,5]$. SPARC-expressing endometrial cancer cells activated fibroblasts only in the presence of FN1, which was abundantly secreted from the cancer cells. SPARC and FN1 were suggested to cooperatively activate fibroblasts. These results suggested that a SPAR C-fibronectin-mediated activation of fibroblasts might be involved in enhanced mobility and invasion of cancer cells.

\section{Supplementary Information}

The online version contains supplementary material available at https://doi. org/10.1186/s12885-021-07875-9.

\footnotetext{
Additional file $\mathbf{1}$ Table S1. Age of patients, from which NF were isolated. Table S2. Clinical characteristics of samples, from which CAF were isolated.
}

Additional file 2 Fig. S1. Full-length blot images of Fig. 1B. Additional file $\mathbf{3}$ Fig. S2. Full-length blot images of Fig. 2A. Additional file $\mathbf{4}$ Fig. S3. Full-length blot images of Fig. 2D Additional file 5 Fig. S4. Full-length blot images of Fig. $3 \mathrm{~A}$. Additional file 6 Fig. S5. Full-length blot images of Fig. 3B. Additional file 7 Fig. S6. Full-length blot images of Fig. 4B. Additional file 8 Fig. S7. Full-length blot images of Fig. 5B. Additional file 9 Fig. S8. Full-length blot images of Fig. $5 \mathrm{C}$. Additional file 10 Fig. S9. Full-length blot images of Fig. 5F. Additional file 11 Fig. S10. Full-length blot images of Fig. 6B. Additional file 12 Fig. S11. Full-length blot images of Fig. $6 \mathrm{~F}$.

\begin{abstract}
Abbreviations
CAF: cancer-associated fibroblasts; EC: endometrial cancer; EMT: epithelial-tomesenchymal transition; NF: normal fibroblasts; RT-qPCR: reverse transcription quantitative polymerase chain reaction

\section{Acknowledgements}

We appreciate the technical support by Sawako Adachi and the Research Support Center, Graduate School of Medical Science, Kyushu University for technical support. We thank Dr. Yumi Nagata at the IVF Nagata Clinic for providing us endometrial stromal fibroblasts. We also thank Dr. Takako Sasaki at Oita University for providing the FLAG-tagged SPARC plasmid. We thank Gabrielle White Wolf, PhD, from Edanz Group (https://en-author-services. edanzgroup.com/ac) for editing a draft of this manuscript.
\end{abstract}

Authors' contributions

KA and KK designed the work. SY and EH carried out functional assays and SY, KA and KK wrote the essay and revised it. SY and KA analyzed statistic 
data. SY, KA, HY1, IO, YM, KO, HY2 and KK interpreted the data. All the authors read and approved the final manuscript. We thank the individual contribution of Ming Li.

\section{Funding}

This work was supported by JSPS KAKENHI Grant Number 15H01517, 15 K15608 (funding to KK), 26462529, 17 K11280 (funding to KA) the FUKUOKA OBGYN Researcher's Charity Foundation Fund, Japan (funding to SY). All materials in in vitro experiments and for expression analysis were financed by the above funders.

\section{Availability of data and materials}

All data generated or analyzed during this study are included in this published article.

\section{Ethics approval and consent to participate}

Written informed consent was obtained from all patients. The study was approved by the Ethical Committee of Kyushu University.

\section{Consent for publication}

Not applicable.

\section{Competing interests}

The authors have no conflict of interest.

\section{Received: 20 September 2020 Accepted: 3 February 2021}

\section{Published online: 12 February 2021}

\section{References}

1. Nagaraju GP, Dontula R, El-Rayes BF, et al. Molecular mechanisms underlying the divergent roles of SPARC in human carcinogenesis. Carcinogenesis. 2014;35:967-73

2. Wong SL, Sukkar MB. The SPARC protein: an overview of its role in lung cancer and pulmonary fibrosis and its potential role in chronic airways disease. Br J Pharmacol. 2017:174:3-14.

3. Robert G, Gaggioli C, Bailet O, et al. SPARC represses E-cadherin and induces mesenchymal transition during melanoma development. Cancer Res. 2006; 66:7516-23.

4. Fenouille $\mathrm{N}$, Tichet $\mathrm{M}$, Dufies $\mathrm{M}$, et al. The epithelial-mesenchymal transition (EMT) regulatory factor SLUG (SNAI2) is a downstream target of SPARC and AKT in promoting melanoma cell invasion. PLoS One. 2012;7:e40378.

5. Hung JY, Yen MC, Jian SF, et al. Secreted protein acidic and rich in cysteine (SPARC) induces cell migration and epithelial mesenchymal transition through WNK1/snail in non-small cell lung cancer. Oncotarget. 2017;8: 63691-702.

6. Brown TJ, Shaw PA, Karp X, et al. Activation of SPARC expression in reactive stroma associated with human epithelial ovarian cancer. Gynecol Oncol. 1999:75:25-33.

7. Rodriguez-Jimenez FJ, Caldes T, Iniesta P, et al. Overexpression of SPARC protein contrasts with its transcriptional silencing by aberrant hypermethylation of SPARC CpG-rich region in endometrial carcinoma. Oncol Rep. 2007:17:1301-7.

8. Sato N, Fukushima N, Maehara N, et al. SPARC/osteonectin is a frequent target for aberrant methylation in pancreatic adenocarcinoma and a mediator of tumor-stromal interactions. Oncogene. 2003;22:5021-30.

9. Shin M, Mizokami A, Kim J, et al. Exogenous SPARC suppresses proliferation and migration of prostate cancer by interacting with integrin beta1. Prostate. 2013;73:1159-70.

10. Infante JR, Matsubayashi $\mathrm{H}$, Sato N, et al. Peritumoral fibroblast SPARC expression and patient outcome with resectable pancreatic adenocarcinoma. J Clin Oncol. 2007;25:319-25.

11. Sangaletti S, Stoppacciaro A, Guiducci C, et al. Leukocyte, rather than tumorproduced SPARC, determines stroma and collagen type IV deposition in mammary carcinoma. J Exp Med. 2003;198:1475-85.

12. Brekken RA, Puolakkainen P, Graves DC, et al. Enhanced growth of tumors in SPARC null mice is associated with changes in the ECM. J Clin Invest. 2003; 111:487-95.

13. Puolakkainen PA, Brekken RA, Muneer S, et al. Enhanced growth of pancreatic tumors in SPARC-null mice is associated with decreased deposition of extracellular matrix and reduced tumor cell apoptosis. Mol Cancer Res. 2004;2:215-24.
14. Sangaletti S, Di Carlo E, Gariboldi S, et al. Macrophage-derived SPARC bridges tumor cell-extracellular matrix interactions toward metastasis. Cancer Res. 2008;68:9050-9.

15. Arnold SA, Rivera LB, Miller AF, et al. Lack of host SPARC enhances vascular function and tumor spread in an orthotopic murine model of pancreatic carcinoma. Dis Model Mech. 2010;3:57-72.

16. Said N, Najwer I, Motamed K. Secreted protein acidic and rich in cysteine (SPARC) inhibits integrin-mediated adhesion and growth factor-dependent survival signaling in ovarian cancer. Am J Pathol. 2007;170:1054-63.

17. Said N, Frierson HF, Sanchez-Carbayo M, et al. Loss of SPARC in bladder cancer enhances carcinogenesis and progression. J Clin Invest. 2013;123:751-66.

18. Aycock RL, Bradshaw AC, Sage EH, et al. Development of UV-induced squamous cell carcinomas is suppressed in the absence of SPARC. J Invest Dermatol. 2004;123:592-9.

19. Sansom OJ, Mansergh FC, Evans MJ, et al. Deficiency of SPARC suppresses intestinal tumorigenesis in APCMin/+ mice. Gut. 2007;56:1410-4.

20. Wong SY, Crowley D, Bronson RT, et al. Analyses of the role of endogenous SPARC in mouse models of prostate and breast cancer. Clin Exp Metastasis. 2008:25:109-18.

21. Sage EH, Reed M, Funk SE, et al. Cleavage of the matricellular protein SPARC by matrix metalloproteinase 3 produces polypeptides that influence angiogenesis. J Biol Chem. 2003;278:37849-57.

22. Podgorski I, Linebaugh $B E_{1}$ Koblinski JE, et al. Bone marrow-derived cathepsin $\mathrm{K}$ cleaves SPARC in bone metastasis. Am J Pathol. 2009;175:1255-69.

23. Clark CJ, Sage EH. A prototypic matricellular protein in the tumor microenvironment--where there's SPARC, there's fire. J Cell Biochem. 2008; 104:721-32.

24. Yusuf $N$, Inagaki $T$, Kusunoki $\mathrm{S}$, et al. SPARC was overexpressed in human endometrial cancer stem-like cells and promoted migration activity. Gynecol Oncol. 2014;134:356-63.

25. Tomari H, Kawamura T, Asanoma K, et al. Contribution of senescence in human endometrial stromal cells during proliferative phase to embryo receptivityt. Biol Reprod. 2020;103:104-13.

26. Zhang N, Wang Y, Liu H, et al. Extracellular vesicle encapsulated microRNA320a inhibits endometrial cancer by suppression of the HIF1a/VEGFA axis. Exp Cell Res. 2020:394:112113.

27. Aprelikova O, Palla J, Hibler B, et al. Silencing of miR-148a in cancerassociated fibroblasts results in WNT10B-mediated stimulation of tumor cell motility. Oncogene. 2013;32:3246-53.

28. Aprelikova O, Yu X, Palla J, et al. The role of miR-31 and its target gene SATB2 in cancer-associated fibroblasts. Cell Cycle. 2010;9:4387-98.

29. Subramaniam KS, Tham ST, Mohamed Z, et al. Cancer-associated fibroblasts promote proliferation of endometrial cancer cells. PLoS One. 2013;8:e68923.

30. Teng F, Tian WY, Wang YM, et al. Cancer-associated fibroblasts promote the progression of endometrial cancer via the SDF-1/CXCR4 axis. J Hematol Oncol. 2016:9:8

31. Ohmaru-Nakanishi T, Asanoma K, Fujikawa M, et al. Fibrosis in Preeclamptic placentas is associated with stromal fibroblasts activated by the transforming growth factor- $\beta 1$ signaling pathway. Am J Pathol. 2018;188: 683-95

32. Asanoma K, Liu G, Yamane T, et al. Regulation of the mechanism of TWIST1 transcription by BHLHE40 and BHLHE41 in Cancer cells. Mol Cell Biol. 2015; 35:4096-109.

33. Kalluri R. The biology and function of fibroblasts in cancer. Nat Rev Cancer. 2016:16:582-98

34. Shi Q, Bao S, Maxwell JA, et al. Secreted protein acidic, rich in cysteine (SPARC), mediates cellular survival of gliomas through AKT activation. J Biol Chem. 2004;279:52200-9.

35. Shi Q, Bao S, Song $L$, et al. Targeting SPARC expression decreases glioma cellular survival and invasion associated with reduced activities of FAK and ILK kinases. Oncogene. 2007;26:4084-94.

36. Koukourakis MI, Giatromanolaki A, Brekken RA, et al. Enhanced expression of SPARC/osteonectin in the tumor-associated stroma of non-small cell lung cancer is correlated with markers of hypoxia/acidity and with poor prognosis of patients. Cancer Res. 2003;63:5376-80.

37. Mantoni TS, Schendel RR, Rodel F, et al. Stromal SPARC expression and patient survival after chemoradiation for non-resectable pancreatic adenocarcinoma. Cancer Biol Ther. 2008;7:1806-15.

38. Chlenski A, Liu S, Guerrero LJ, et al. SPARC expression is associated with impaired tumor growth, inhibited angiogenesis and changes in the extracellular matrix. Int J Cancer. 2006;118:310-6. 
39. Sangaletti S, Tripodo C, Santangelo A, et al. Mesenchymal transition of highgrade breast carcinomas depends on extracellular matrix control of myeloid suppressor cell activity. Cell Rep. 2016;17:233-48.

40. Zhu J, Wang LY, Li CY, et al. SPARC promotes self-renewal of limbal epithelial stem cells and ocular surface restoration through JNK and p38MAPK signaling pathways. Stem Cells. 2020;38:134-45.

41. Sharma S, Xing F, Liu Y, et al. Secreted protein acidic and rich in cysteine (SPARC) mediates metastatic dormancy of prostate Cancer in bone. J Biol Chem. 2016;291:19351-63.

42. Salzer MC, Lafzi A, Berenguer-Llergo A et al. Identity Noise and Adipogenic Traits Characterize Dermal Fibroblast Aging. Cell. 2018; 175: 1575-1590. e1522.

43. Neumann E, Riepl B, Knedla A, et al. Cell culture and passaging alters gene expression pattern and proliferation rate in rheumatoid arthritis synovial fibroblasts. Arthritis Res Ther. 2010;12:R83.

44. Dawson $\mathrm{K}, \mathrm{Wu} \mathrm{CT}$, Qi XY, et al. Congestive heart failure effects on atrial fibroblast phenotype: differences between freshly-isolated and cultured cells. PLoS One. 2012;7:e52032.

45. Bauer M, Su G, Casper C, et al. Heterogeneity of gene expression in stromal fibroblasts of human breast carcinomas and normal breast. Oncogene. 2010; 29:1732-40.

46. Kooistra A, Elissen NM, König JJ, et al. Immunocytochemical characterization of explant cultures of human prostatic stromal cells. Prostate. 1995;27:42-9.

47. Kusunoki S, Kato K, Tabu K, et al. The inhibitory effect of salinomycin on the proliferation, migration and invasion of human endometrial cancer stem-like cells. Gynecol Oncol. 2013;129:598-605.

48. Barker TH, Baneyx G, Cardo-Vila M, et al. SPARC regulates extracellular matrix organization through its modulation of integrin-linked kinase activity. J Biol Chem. 2005;280:36483-93.

49. Golembieski WA, Thomas SL, Schultz CR, et al. HSP27 mediates SPARCinduced changes in glioma morphology, migration, and invasion. Glia. 2008; 56:1061-75.

50. Alam R, Schultz CR, Golembieski WA, et al. PTEN suppresses SPARC-induced PMAPKAPK2 and inhibits SPARC-induced Ser78 HSP27 phosphorylation in glioma. Neuro-Oncology. 2013;15:451-61.

51. Nagaraju GP, Sharma D. Anti-cancer role of SPARC, an inhibitor of adipogenesis. Cancer Treat Rev. 2011;37:559-66.

52. Murphy-Ullrich JE. The de-adhesive activity of matricellular proteins: is intermediate cell adhesion an adaptive state? J Clin Invest. 2001:107:785-90.

53. Levental $K R$, Yu H, Kass $L$, et al. Matrix crosslinking forces tumor progression by enhancing integrin signaling. Cell. 2009;139:891-906.

54. Ng MR, Brugge JS. A stiff blow from the stroma: collagen crosslinking drives tumor progression. Cancer Cell. 2009;16:455-7.

55. Girotti MR, Fernandez M, Lopez JA, et al. SPARC promotes cathepsin Bmediated melanoma invasiveness through a collagen $1 /$ alpha2beta1 integrin axis. J Invest Dermatol. 2011;131:2438-47.

56. Nakamura K, Yamanouchi K, Nishihara M. Secreted protein acidic and rich in cysteine internalization and its age-related alterations in skeletal muscle progenitor cells. Aging Cell. 2014;13:175-84.

\section{Publisher's Note}

Springer Nature remains neutral with regard to jurisdictional claims in published maps and institutional affiliations.

Ready to submit your research? Choose BMC and benefit from:

- fast, convenient online submission

- thorough peer review by experienced researchers in your field

- rapid publication on acceptance

- support for research data, including large and complex data types

- gold Open Access which fosters wider collaboration and increased citations

- maximum visibility for your research: over $100 \mathrm{M}$ website views per year

At $\mathrm{BMC}$, research is always in progress.

Learn more biomedcentral.com/submissions 